\title{
Inverse Brillouin Function and Demonstration of Its Application
}

\author{
Aleksandr Hayrapetyan \\ Faculty of Physics, Yerevan State University, Armenia
}

\section{Abstract}

The Brillouin function arises in the quantum theory of paramagnetic materials, where it describes the dependence of the magnetization on the externally applied magnetic field and on the temperature of the system. There is no closed form exact analytical expression for the inverse Brillouin function, however, there have been several approximations proposed. In this work, we first compare relative errors and simplicity of several approximations for the inverse Brillouin function. Next, we demonstrate the application of the inverse Brillouin function by determining the Hamiltonian of the system using the simulation data of the magnetization dependence on the temperature. Then we compare the Hamiltonian that was used to set up the simulation with the Hamiltonian determined from the magnetization temperature dependence and an approximation to the inverse Brillouin function. We found that some of the approximations for the inverse Brillouin function can be used to accurately predict the Hamiltonian of the system given the magnetization dependence on temperature.

Keywords: inverse, brillouin, function, demonstration, application

\section{Introduction}

To study different paramagnetic phenomena in the classical and quantum theory of paramagnetism, two special functions are used. The first of these is the Langevin function [1]. This function appears when calculating the magnetization if there is a medium in which $\mathrm{N}$ atoms is contained in a unit volume. Magnetization takes place in cases where atoms have magnetic moments $\mu$ that are oriented to an external magnetic. Several mathematical calculations will be shown in the next section of this paper.

Another special function, which is applied while considering various problems related to the quantum theory of paramagnetism, is the Brillouin function [1]. In the contemporary professional literature not only the direct Brillouin function is used, but also its inverse function. These functions are normally mentioned in the following contexts: polymer science, molecular dynamics modeling, magnetism, rubber theory, etc. But unfortunately, the inverse Brillouin and Langevin functions cannot be calculated 
analytically, so their approximations are taken, in which the relative error tends to the minimum.

It is noteworthy to mention a few words about paramagnetism, in which we use the above-mentioned special functions. These atoms and molecules have an uncompensated spin magnetic moment. Other examples include free atoms and ions with incomplete inner shells, some molecules with an even number of electrons, lattice defects with an odd number of electrons, metals, other types of atoms and molecules. For the first time, the theory of paramagnetic susceptibility was created by P. Langevin and was used in the above mentioned problem.

In modern literature, one may find the inverse Langevin function, has a wide range of uses. But the quest for the inverse Brillouin function, has not yet been crowned with success. Currently, the existing approximations of the inverse Brillouin function are being improved every day. In the sections below, we consider the Langevin and Brillouin functions, their existing approximations, their negative and positive sides, and their applications.

I would like to mention some works related to the above-mentioned functions, their inverse functions and their areas of application. These topics were discussed in the works of the following authors: A.S. Arrott [2], Martin Kröger [3], M.I. Darby [4], and others whose research we will quote in this paper.

At the end of this paper, a inference will be made about all the above mentioned in very detail, which will allow us to contemplate the indicated functions from new perspectives.

Brillouin and Langevin functions and their applications

Langevin function, it's inverse function and their applications

Let's recall the above mentioned task. Suppose we have an environment that contains $\mathrm{N}$ molecules per unit volume. Magnetization arises only when the magnetic moments are oriented under the action of an external field. The energy $U$, which describes the interaction of magnetic moments $(\mu)$ with external field (B), is described by the following scalar product (1):

$U=-\boldsymbol{\mu} * \boldsymbol{B}$

This scalar product can be represented as follows (2):

$U=-\mu B \cos \theta$

where $\theta$ is the angle between the moment vector and the direction of the field.

Within this problem, the field magnetization is calculated by the following equation (3) $M=N \mu\langle\cos \theta\rangle$

where $\mathrm{N}$, as mentioned above, is the concentration, $\langle\cos \theta\rangle$ is the average value of $\cos$ 
$\theta$ taken from the spatial distribution of magnetic moments in the state of thermal equilibrium.

If in this problem we use the Boltzmann distribution, then we can count the probability that the moment of the molecule is located in an element of a given solid angle $d \Omega$. This probability is proportional to $\exp \left(-U / k_{B} T\right)$, and therefore the average value of $\cos \theta$ ( $\langle\cos \theta\rangle$ ) can be calculated by the following equation (4)

$\langle\cos \theta\rangle=\left(\int e^{-\beta U} \cos \theta d \Omega\right) *\left(\int e^{-\beta U} d \Omega\right)^{-1}$

here, $\beta \equiv 1 / k_{B} T$ designation is inserted, and $d \Omega=\sin \theta d \varphi d \theta$. In the above equation, integration is performed over all values of $\varphi$ and over all values of $\theta$. For all values of $\varphi$, we get $2 \pi$, a $\theta$ varies from 0 to $\pi(5)$ :

$\langle\cos \theta\rangle=\frac{\int_{0}^{\pi} 2 \pi \sin \theta \cos \theta \mathrm{e}^{\beta \mu B \cos \theta} d \theta}{\int_{0}^{\pi} 2 \pi \sin \theta \mathrm{e}^{\beta \mu B \cos \theta} d \theta}$

For simplicity, let's introduce the following reductions (6)

$$
s \equiv \cos \theta, x \equiv \frac{\mu B}{k_{B} T}
$$

and after integration we get the following equation (7)

$$
\begin{gathered}
\langle\cos \theta\rangle=\left(\int_{-1}^{1} \exp (s x) s d s\right) *\left(\int_{-1}^{1} \exp (s x) d s\right)^{-1}= \\
=\frac{d}{d x} \ln \int_{-1}^{1} \exp (s x) d s=\frac{d}{d x} \ln (\exp (x)-\exp (-x))-\frac{d}{d x} \ln (x)=\operatorname{cth} x-\frac{1}{x} \equiv L(x)
\end{gathered}
$$

The resulting function is called the Langevin function. It has the following form (8)

$L(x)=\operatorname{cth}(x)+\frac{1}{x}$

The final calculation of the magnetization field is made by the following formula (9)

$M=N \mu L(x)=N \mu\left(\operatorname{cth}(x)+\frac{1}{x}\right)$

This function can be regarded in two cases. When $\mu B \gg k_{B} T$ and when $\mu B \ll k_{B} T$. In the first case, saturation can be found in the graph of the function, and the second case is of great interest for experimenters when calculating various kinds.

Let's look at some approximations of the Langevin function.

If in the Langevin function we expand in the series $c t h(x)$ then for small values of $\mathrm{x}$ we get the following expression (10)

$\operatorname{cth}(x) \approx \frac{1}{x}+\frac{x}{3}-\frac{x^{3}}{45}+\cdots$ 
Putting this expression into the Langevin function, we obtain the following approximation (11)

$L(x) \approx \frac{x}{3}$

For magnetization, we obtain the following expression (12)

$M \approx \frac{N \mu^{2} B}{3 k_{B} T}=\frac{C}{T} B$

Where C stands for Curie (13)

$C=\frac{N \mu^{2}}{3 k_{B}}$

Let's discuss another approximation of the Langevin function. If we decompose the Langevin function in a Taylor series for small values of $x$, we get the following result (15)

$L(x)=\frac{1}{3} x-\frac{1}{45} x^{3}+\frac{2}{945} x^{5}-\frac{1}{4725} x^{7}+\cdots$

This approximation method is an alternative approximation of the $\operatorname{cth}(x)$ approximation. From the point of view of numerical calculation, both approximations are equivalent to each other and are more demanded than direct evaluation of an analytical expression.

It can be said that the Langevin function has been fully investigated and is widely used in describing various tasks.

Below we will discuss the Brillouin function.

Brillouin function, it's inverse function and their applications

Brillouin function, inverse function and its applications

We shall first and foremost recall the formula (15)

$B_{J}(x)=\frac{2 J+1}{2 J} \operatorname{coth}\left(\frac{2 J+1}{2 J} x\right)-\frac{1}{2 J} \operatorname{coth}\left(\frac{1}{2 J} x\right)$

In which $J$ is a positive integer or half-integer number,

$x$ stands for the relation of Zeeman energy ratio of the magnetic moment in an internal field to the heat energy: (16)

$x=\frac{g \mu_{B} J B}{k_{B} T}$

in which $g$ is factor $g, \mu_{B}$ is Bohr magneton, $J$ is the full angular momentum, $B$ is the applied magnetic field, $k_{B}$ is Boltzmann constant, and $T$ is the temperature.

Brillouin function changes by taking values from -1 to 1 , reaching +1 if $x \rightarrow \infty$ and -1 if $x \rightarrow \infty$. Brillouin function has a wide range of utilization, but most often it is used for calculating the magnetization of an ideal paramagnet. It describes the dependency of $M$ 
from the applied magnetic field B and the total angular momentum $J$. Magnetization is expressed through the formulum (17)

$M=N g \mu_{B} J B_{J}(x)$

in which $N$ stands for the quantity of the atoms per unit volume. The Brillouin function turns into that of Langevin in the range of $J \rightarrow \infty$ (8). If $x \rightarrow \infty$, then $B_{J}(x) \rightarrow 1$. This means that the magnetization gets saturated and the magnetic moments become totally aligned in the direction of the applied field. (18)

$M=N g \mu_{B} J$

And now we shall talk about how one should find the inverse function of Brillouin. It is impossible to achieve analytically, but one can throw some approximations apropos the $\operatorname{coth}(x)$ function and find the Brillouin inverse function with some accuracy. If the error of this accuracy does not exceed the applied limit ( $\sim 5 \%)$, then the given approximation can be safely used in different calculations.

The first approximation can be done in case if $J=1 / 2$, and then $B_{J}(x) \rightarrow \tanh (x)$. If we are to take this approximation into account, then we can safely write the following expression (19)

$$
B_{J}(x) \approx \tanh (n x)
$$

in which $n$ is the coefficient dependent solely on $J$. The absolute and relative mistakes depend on this coefficient. We shall turn to the works of Jeno Takacs in order to demonstrate the most precise result possible [5]. Here we have a result which is currently seen as the most precise and recent one, and it is presented as follows (20)

$n \approx \frac{1}{2,667 J}+0.2$

Given the value of $n$, the relative miscalculation makes about $4 \%$. If we are to use the given approximation for $B_{J}(x)$, then we will get the following result for the inverse function [5] (21)

$B_{j}(x)^{-1} \approx \frac{a x J^{2}}{1-b x^{2}}$

$a$ and $b$ are the coefficients that have the following form (22)

$a=\frac{0.5(1+2 J)(1-0.055)}{J-0.27}+\frac{0.1}{J^{2}} \quad b=0.8$

$J$ changes from 1 to 10 . Model (21) is still relevant outside of these values, but with much less accuracy which means that the error value increases.

Let us discuss yet another version of Brillouin inverse function, and then we shall further discuss its use. This other version of Brillouin inverse function reappears when $J=1 / 2$ which resembles the original function by its graph and predicts a quite low range of 
miscalculation. It is as follows: (23) [6]

$B_{J}(x)^{-1} \approx \ln \left(\frac{1+2 s}{1-2 s}\right)$

$s$ stands for magnetization. $s$ changes from 0 to $1 / 2$. This function cannot be used for other values, as there is a logarithm in the function: when the numerator has a negative sign, the function loses its physical meaning.

Now let us discuss its use. The Brillouin inverse function is used in non-iterative mean field theory. Thus, the mean field equation for an arbitrary isotropic spin Hamiltonian of infinite range will be as follows (24)

$s=\sigma B_{J}\left(-\beta \sigma \frac{\partial H}{\partial s}\right) ; \quad \beta=\frac{1}{k_{B} T}$

The given equation can be rewritten in the wollowing manner (25)

$k T=-\frac{\frac{\partial H}{\partial s}}{B_{J}(x)^{-1}}$

One can calculate the dependence of $\mathrm{T}$ from $\mathrm{s}$ using this equation.

The magnetic entropy can be presented in the following form using the Brillouin inverse function (26)

$\Delta S=-k_{B} \int_{0}^{S} B_{J}\left(s^{\prime}\right)^{-1} d s^{\prime}$

$\Delta S$ is to represent the decrease in entropy relative to the paramagnetic phase.

\section{Conclusion}

To sum up, we can claim that the Brillouin inverse function is hard to compute in mathematical calculations. Various approximations are put forward in order to overcome this problem. We have mentioned some of them above. One of these is the approximation $\tanh (x)$ (19), and another one is the approximation $\ln (x)$ (23). In the approximation $\tanh (x)$, the obtained function works with a small admissible error in practice. In the approximation, the obtained function in practice works with a small admissible error. The proof of that is the fact that $B_{J}(x)$ moves toward $\tanh (x)$ when $J=1 / 2$.

The analytical form of Brillouin inverse function has not yet been found. The abovementioned approximate Brillouin inverse functions work within the operating limits of the quantum number of angular momentum. The majority of materials have $J$ from 1 to 10 in engineering practice. This is why many approximations of the Brillouin inverse function have a great demand in current studies.

\section{Acknowledgments}

I would like to thank Rafayel Petrosyan for valuable discussions. 


\section{References}

[1] C. Kittel ,1963, Introduction to Solid State Physics, 4th edition (New York: John Wiley and Sons, Inc.), pp. 518-523.

[2] A. S. Arrott, J. Appl. Phys. 103, 07C715 (2008).

[3] M. Kröger, Simple, admissible, and accurate approximants of the inverse Langevin and Brillouin functions, relevant for strong polymer deformations and flows, J.

NonNewtonian Fluid Mec. 223 (2015) pp. 77-87.

[4] Darby, M.I. (1967). "Tables of the Brillouin function and of the related function for the spontaneous magnetization". Br. J. Appl. Phys. 18 (10): 1415-1417

[5] Takacs, Jeno (2016). "Approximations for Brillouin and its reverse function". COMPEL. 35 (6): 2095.

[6] J. Katriel Continued-fraction approximation for the Brillouin function Phys. Stat. Sol., 189 (1987), p. 308 